\title{
Quality of life differences between African Americans and Hispanic Americans with multiple sclerosis
}

\author{
Diamond Garcia, Jason Ledesma, Kristen Berube, Sarah Valdez, Eric Tamrazian and Bijal Mehta*
}

\begin{abstract}
Background: In comparison to non-minority patients with multiple sclerosis, minority research in multiple sclerosis continues to be only a few percent of the literature. Often, the comparator group is with the Caucasian populations of the world, who themselves comprise of non-ubiquitous groups. Comparisons between minority groups have not been done as they relate to quality of life, partly because the populations are smaller. Our study will compare the quality life differences between two minority populations living in the same geographic and urban area.
\end{abstract}

Methods: This study utilized cross-sectional data from an observational prospective cohort study. A total of 28 minority patients were included in this study. All patients were on a disease modifying therapy. Demographic information was obtained. The primary outcome measure was the MSQLI and all scores were derived from the MSQLI user's manual.

Results: The MSQLI scores of the Mental Health Index were lower in all areas for Hispanic White MS patients as compared to Non-Hispanic African American patients, but 3 of 5 were statistically significant: MHI total score, $\mathrm{MHI}$ anxiety, and MHI behavioral control subscores (all $p<0.05$ ). When investigating if EDSS associated with MSQLI measures, independent of minority group, the only noted difference was between the mild (EDSS $<$ or $=3$ ) and the moderate group (EDSS 3.5-5.5) in the Physical Components Score and Health Transition Score $(p=0.028$ and $p<0.05)$.

Conclusion: The study begins to elucidate differences in quality life measures between minorities with multiple sclerosis potentially leading to culturally competent care.

\section{Key practice points}

1. Quality of Life differences exist between patients based on race, ethnicity, and socioeconomic status

2. Mental health issues of multiple sclerosis patients can vary based of race, ethnicity, and other nondisease based factors in multiple sclerosis patients

3. Clinicians may benefit patients from asking about and potentially addressing their MS patient's quality of life issues

\section{Introduction}

Multiple Sclerosis (MS) is a chronic, inflammatory, demyelinating, and ultimately progressive disease of the central nervous system that causes physical and cognitive

* Correspondence: Bijal@ucla.edu

Department of Neurology, The David Geffen School of Medicine,

Harbor-UCLA Medical Center, Los Angeles Biomedical Institute, Torrance, USA disability and disproportionately affects women $[1,2]$. The cause of MS is still unknown. However, it is believed to be associated with genetic factors and environmental exposures [3]. There is no cure for MS and as a result, treatment modalities are aimed at reducing disease progression and managing symptoms [4]. Symptoms include fatigue, gait imbalance, bowel and bladder dysfunction, visual disturbances, cognitive dysfunction, sexual dysfunction, pain and depression, which negatively affect a patient's health-related quality of life (HRQoL) $[1,2,5]$.

While the physical manifestations of MS are assessed using the Kurtzke Expanded Disability Status Scale (EDSS), the psychological manifestations are evaluated by a patient's health-related quality of life (HRQoL). The EDSS is the most common outcome measure of impairment/disability for MS patients [6] and scores range from 0 to 10 in 0.5 increments with higher scores indicating higher levels of disability. On the other hand, the 
HRQoL requires a broader measure of disease burden compared to physical impairment and disability level alone [7]. The HRQoL aims to measure a person's more comprehensive well-being including physical, mental and social health [7].

Previous studies have suggested a higher correlation of MS with those of European descent [8, 9]. However, in a 2015 article published in Neurology Clinical Practice, Khan and colleagues revealed that out of 60,000 published articles on MS, only 113 focused on African-Americans (about $0.20 \%$ ) and even less, 23, focused on Hispanic Americans (about .040\%) with MS [10]. The AfricanAmerican population in the United States (U.S) is projected to rise from $13.3 \%$ in 2016 to $17.9 \%$ in 2060 $[11,12]$. The Hispanic population in the U.S is projected to rise from $17.8 \%$ in 2016 to $28.6 \%$ in 2060 [11, 12]. African-Americans and Hispanics make up the two largest minority groups in the U.S, but only represented less than $1 \%$ of the published articles on MS. African-Americans and Hispanics with MS are understudied [10]. However, in the past decade MS literature has shown an increased interest in MS health in ethnic populations [10].

With the importance of HRQoL in MS and AfricanAmericans and Hispanics being underrepresented in MS literature, we compared the HRQoL differences between two understudied minority populations in a large urban area. The primary objective of this analysis was to examine the differences in HRQoL between Non-Hispanic AfricanAmericans and Hispanic White MS patients using the HRQoL data from an observational prospective cohort study. The secondary objective of this analysis was to compare the HRQoL data to the EDSS scores, age and sex of these racial and ethnic minority MS patients.

\section{Methods}

\section{Study population}

This study used cross-sectional data from an observational prospective cohort study $(N=46)$ comparing the level of vitamin A in relapsing remitting (RRMS) and secondary progressive Multiple Sclerosis (SPMS) patients. Subjects for this study were individuals with a diagnosis of RRMS or SPMS and with normal vitamin D levels $(38-98 \mathrm{mcg} /$ $\mathrm{dL}$ ) or supplemented with vitamin $\mathrm{D}$ to reach a normal level. Study participants spoke either English and/or Spanish. They also had to be currently taking a disease modifying therapy or MS treatment and willing to participate in an MRI at our facility every 6 months. Of the total 46 patients, 28 met the criteria for inclusion. This research has been reviewed and approved by a Human Subjects Committee (IRB) at the Los Angeles Biomedical Institute.

\section{Demographic and MS characteristics measures}

Demographic information such as age, sex, language, race/ethnicity, education level, marital status, employment status, use of smoking tobacco, alcohol use, illicit drug user and the number of comorbidities were collected at baseline. Clinical MS characteristics such as type of MS and year of MS diagnosis were also collected at baseline.

\section{Primary outcome measure}

HRQoL was measured using the MSQLI and all scores were derived using the MSQLI user's manual [13]. The MSQLI is a comprehensive patient reported HRQoL measure specifically tailored for MS patients [13]. It is made up of 10 components: 1) Generic Quality of Life Measure: Short Form Health Status Questionnaire (SF-36), 2) Modified Fatigue Impact Scale (MFIS), 3) MOS Pain Effects Scale (PES), 4) Sexual Satisfaction Scale (SSS), 5) Bladder Control Scale (BLCS), 6) Bowel Control Scale (BWCS), 7) Impact of Visual Impairment Scale (IVIS), 8) Perceived Deficits Questionnaire (PDQ), 9) Mental Health Inventory (MHI), 10) MOS Modified Social Support Survey (MSSS) [13, 14]. The SF-36 generates two generic summary scores, the Physical Components Summary Scale (PCS) and the Mental Component Summary Scale (MCS). These 10 components reflect the symptoms that occur most often in MS [13, 14].

Table 1 lists the MSQLI components and scoring scale for reference. The MSQLI was administered by study personnel 6 months from baseline and on the last study visit. For this study, only the 6 month MSQLI data (a single-time point) was analyzed. Of this baseline sample $(N=46), 28$ study participants completed the MSQLI. The MSQLI was administered either in-person or overthe-phone in English or Spanish. For the Spanish-speaking study participants, our Spanish-speaking research associates translated the MSQLI to Spanish because no Spanish version of the MSQLI existed.

\section{Secondary outcome measures}

In addition to the MSQLI, an EDSS was administered by a neurologist at each study visit (every 3 months). In this study, the EDSS score closest to the MSQLI administered date was used to compare to the 10 MSQLI components. EDSS scores were distributed into 3 categories: mild disability represented by an EDSS score of $\leq 3$, mild-moderate disability represented by an EDSS score of 3.5-5.5 and moderate-severe disability represented by an EDSS score $>5.5$ (patients requiring assistance or assistive devices for walking). The EDSS scores of each group were also compared to eliminate disability level as a possible causation of the significant MSQLI differences found between groups. Moreover, demographic information such as ethnicity-race, age and sex of the 28 study participants were compared to the MSQLI data. For the ethnicity-race comparison, only the MSQLI results of Non-Hispanic African-American and Non-White Hispanic study participants were compared. Non-Hispanic White 
Table 1 MSQLI Measure and Scoring

\begin{tabular}{|c|c|c|}
\hline Outcome & Measure & Scale \\
\hline SF-36 HT & single item covering change in health status over the last year & $1-5^{a}$ \\
\hline SF-36 PCS & generic physical components summary scale & $0-100^{b}$ \\
\hline SF-36 MCS & generic mental component summary scale & $0-100^{b}$ \\
\hline MFIS & impact of fatigue on a patient's activities & $0-84^{c}$ \\
\hline PES & impact of pain on a patient's mood and behavior & $6-30^{c}$ \\
\hline SSS & sexual satisfaction problems & $4-24^{c}$ \\
\hline BLCS & bladder control problems & $0-22^{c}$ \\
\hline BWCS & bowel control problems & $0-26^{c}$ \\
\hline IVIS & impact of visual problems & $0-15^{\mathrm{C}}$ \\
\hline PDQ & perceived cognitive impairment & $0-80^{c}$ \\
\hline MHI Total & overall emotional functioning & $0-100^{d}$ \\
\hline MHI Anxiety & anxiety presence & $0-100^{d}$ \\
\hline MHI Depression & depression presence & $0-100^{d}$ \\
\hline MHI Behavioral & the ability to control one's behaviors & $0-100^{d}$ \\
\hline \multicolumn{3}{|l|}{ Control } \\
\hline MHI Positive Affect & experience of positive moods & $0-100^{d}$ \\
\hline MSSS & perceived social support & $0-100^{c}$ \\
\hline
\end{tabular}

Abbreviations: MSQLI multiple sclerosis quality of life inventory, SF-36 36-item short form generic health status questionnaire, $H T$ health transition item, $P C S$ physical components summary scale, MCS mental component summary scale, MFIS modified fatigue impact scale, $P E S$ MOS pain effects scale, SSS sexual satisfaction scale, BLCS bladder control scale, BWCS bowel control scale, IVIS impact of visual impairment scale, $P D Q$ perceived deficits questionnaire, MHI mental health inventory, MSSS modified social support survey

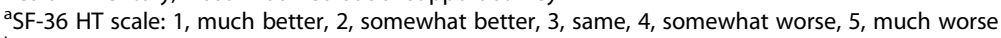

${ }^{\mathrm{b}}$ Higher scores indicate better health

cHigher scores indicate greater amount of measure

${ }^{\mathrm{d}}$ Higher scores indicate better mental health

study participants and those of Asian descent were excluded from the analysis due to low subject numbers. For the age category, study participants were divided into 3 age groups: the youngest group representing those born between 1980 and 1994 (ages 23-37), the mid-aged group representing those born between 1965 and 1979 (ages 3852 ), and the older group representing those born between 1950 and 1964 (ages 53-67).

\section{Statistical analysis}

Descriptive statistics were used to describe the sample and overall data. In order to analyze the MSQLI numerical data by ethnicity-race, EDSS scores, age and sex, a Student's t-test was performed to compare the means between two groups. A two-tailed distribution and two samples, unequal variance was used for the Student's t-test. Results with a $P$-value of less than 0.05 were considered significant. For the SF-36 health transition score, the Mann-U Whitney test was used instead due to the data type being of ordinal categorical data. For measures that had more than two groups to compare such as the EDSS scores and age, an Analysis of Variance (ANOVA) was used to compare the means of the multiple groups. For those comparisons that showed a significant difference $(P$-value $\leq 0.5)$, the means of each group were calculated to determine which of the groups contributed to the differences. LibreOffice spreadsheets version 5.3 was used for all statistical tests.

\section{Results}

\section{MSQLI and ethnicity-race}

Twenty-eight patients with MS completed the MSQLI. Demographic and clinical MS characteristics of the patients are given in Table 2. MSQLI scores on the Mental Health Inventory (MHI) measures were lower in all areas for Hispanic Whites compared to Non-Hispanic African Americans. The average scores for the MHI between Non-Hispanic African Americans and Hispanic Whites are shown in Table 3. Although Hispanic Whites presented lower MHI averages, only 3 MHI measures out of 5 were found to be statistically significant: MHI total score, MHI anxiety and behavioral control subscales. Non-Hispanic African Americans indicated a better total mental health score $(84.11$ vs. $69.05, P$-value $=0.018)$, lower anxiety $(89.20$ vs. $63.14, P$-value $=0.001)$ and better behavioral control ( 90.50 vs. $73.57, P$-value $=0.026)$ compared to Hispanic Whites. Interestingly, Non-Hispanic African Americans also demonstrated a higher SF-36 Mental Component Summary Scale (MCS) score (54.48 vs. $49.97, P$-value $=0.392)$ compared to Hispanic Whites 
Table 2 Demographic and clinical characteristics of patients

\begin{tabular}{|c|c|}
\hline Variable & Value \\
\hline Age $^{a}$, years (mean $\pm S D$ ), range & $41.2 \pm 11.4(21-62)$ \\
\hline \multicolumn{2}{|l|}{ Sex, n (\%) } \\
\hline Female & $18(64.3 \%)$ \\
\hline Male & $10(35.7 \%)$ \\
\hline \multicolumn{2}{|l|}{ Language, n (\%) } \\
\hline English & $22(78.6 \%)$ \\
\hline Spanish & $6(21.4 \%)$ \\
\hline \multicolumn{2}{|l|}{ Race/Ethnicity, n (\%) } \\
\hline White/Non-Hispanic & $2(7.1 \%)$ \\
\hline White/Hispanic & $14(50.0 \%)$ \\
\hline African American/Non-Hispanic & $10(35.7 \%)$ \\
\hline Asian/Non-Hispanic & $1(3.6 \%)$ \\
\hline Asian/Hispanic & $1(3.6 \%)$ \\
\hline \multicolumn{2}{|l|}{ Education level, n (\%) } \\
\hline$<$ High school & $7(25.0 \%)$ \\
\hline High school diploma or GED & $6(21.4 \%)$ \\
\hline Associate's Degree (in progress) ${ }^{b}$ & $2(7.1 \%)$ \\
\hline Associate's Degree (completed) ${ }^{b}$ & $2(7.1 \%)$ \\
\hline Bachelor's Degree (in progress) & $3(10.7 \%)$ \\
\hline Bachelor's Degree (completed) & $6(21.4 \%)$ \\
\hline Master's Degree (in progress) & $1(3.6 \%)$ \\
\hline Master's Degree (completed) & $1(3.6 \%)$ \\
\hline \multicolumn{2}{|l|}{ Marital Status, n (\%) } \\
\hline Single & $16(57.1 \%)$ \\
\hline Married & $8(28.6 \%)$ \\
\hline Divorced & $4(14.3 \%)$ \\
\hline \multicolumn{2}{|l|}{ Employment Status } \\
\hline Employed & $11(39.3 \%)$ \\
\hline Unemployed & $11(39.3 \%)$ \\
\hline On Disability or Supplemental Security Income & $6(21.4 \%)$ \\
\hline Current or Former Smoker & $11(39.3 \%)$ \\
\hline Current or Former Alcohol Drinker & $14(50.0 \%)$ \\
\hline Current or Former Illicit Drug User & $12(42.9 \%)$ \\
\hline \multicolumn{2}{|l|}{ Clinical Form of MS, n (\%) } \\
\hline Relapsing-remitting & $26(92.9 \%)$ \\
\hline Secondary progressive & $2(7.1 \%)$ \\
\hline Disease Duration ${ }^{c}$, years (mean $\pm S D$ ), range & $5.5 \pm 4.4,(1-10)$ \\
\hline \multicolumn{2}{|l|}{ Number of Comorbidities } \\
\hline 0 & $15(53.6 \%)$ \\
\hline 1 & $9(32.1 \%)$ \\
\hline 2 & $3(10.7 \%)$ \\
\hline 3 & $1(3.6 \%)$ \\
\hline EDSS score, (mean $\pm S D$ ), range & $4.2 \pm 2.0(1-8)$ \\
\hline
\end{tabular}

Abbreviations: SD standard deviation, GED General Education Diploma, MS multiple sclerosis, EDSS expanded disability status scale aAge when MSQLI was administered

Includes academic, occupational, technical, or vocational programs

'Year of MS diagnosis to MSQLI administered date
Table 3 Average MHI between African Americans and Hispanic Whites

\begin{tabular}{|c|c|c|c|}
\hline Outcome & African American & Hispanic White & $P$-value \\
\hline MHI Total Score ${ }^{a}$ & 84.11 & 69.05 & 0.018 \\
\hline MHI Anxiety ${ }^{a}$ & 89.20 & 63.14 & 0.001 \\
\hline MHI Depression ${ }^{\mathrm{a}}$ & 81.50 & 71.79 & 0.273 \\
\hline MHI Behavioral Control ${ }^{\mathrm{a}}$ & 90.50 & 73.57 & 0.026 \\
\hline MHI Positive Affect ${ }^{a}$ & 75.50 & 67.86 & 0.267 \\
\hline
\end{tabular}

Although not statistically significant, Hispanic Whites scored lower in mental health even among a generic measure of mental health. Furthermore, Hispanic Whites reported less comorbidities than Non-Hispanic African Americans $(0.14$ vs. $1.30, P$-value $=0.001)$. All, but two Hispanic White patients reported 0 comorbidities while all, but one patient of the latter group reported at least 1 comorbidity. The top 3 comorbidities reported were hypertension (high blood pressure), dyslipidemia (high cholesterol) and cardiovascular disease (heart disease) respectively. Hypertension accounted for $50 \%$ of the comorbidities reported including being the only comorbidity reported by the Hispanic White group. Additionally, there was no statistically significant difference found between the EDSS scores of Hispanic Whites and Non-Hispanic African Americans indicating that the statistically significant differences found were independent of the severity of their EDSS based disability. Hispanics with MS had an average EDSS score of 4.21 and African Americans had an average of 4.75. Moreover, Hispanics with MS had an average MS disease duration of 5 years compared to 5.2 years for Non-Hispanic African Americans. There were also no statistically significant differences found between the use of illicit drugs or the use of interferon medications between the two groups, as interferons have been reported to exacerbate depressive symptoms [15]. Of the $42 \%$ of Hispanic Whites and Non-Hispanic African Americans on interferon MS medications, $70 \%$ of those were Hispanic White and 30\% were Non-Hispanic African American. Rebif and Betaseron were tied for the most commonly reported interferon medication. There were no other statistically significant differences found in the remaining MSQLI measures between Non-Hispanic African Americans and Hispanic Whites.

\section{MSQLI and EDSS}

EDSS scores were categorized into 3 groups: mild disability (EDSS score $\leq 3$ ), mild-moderate disability (EDSS score 3.5-5.5) and moderate-severe disability (EDSS score $>5.5$ ). When comparing all the MSQLI measures to the EDSS scores, statistically significant differences were only found between the mild disability group and 
the moderate-severe disability group in the SF-36 Physical Components Summary Scale (PCS) and SF-36 Health Transition (HT) score. The average scores for the SF-36 PCS and SF-36 HT between the mild and moderate-severe disability groups are shown in Table 4. Not surprisingly, patients with an EDSS $\leq 3$ indicated a better physical health score ( 43.56 vs. $33.21, P$-value $=0.028)$ and a better change in health status over the last year (1.56 vs. $2.80, P$-value < 0.05) compared to those with an EDSS score of greater than 5.5. Note, that a higher physical components score corresponds with a better physical health and a lower SF-36 HT score represents a lesser change in health status over the previous year. There were no statistically significant differences found in the remaining MSQLI mean scores between these 3 EDSS groups.

\section{MSQLI and age}

MS patients were divided into 3 age categories: the youngest group representing those born between 1980 and 1994 (ages 23-37), the mid-aged group representing those born between 1965 and 1979 (ages 38-52), and the older group representing those born between 1950 and 1964 (ages 53-67). In terms of the age of the MS patients, significant differences were found in the SF-36 Health Transition (HT), the Modified Fatigue Impact Scale (MFIS) and the Perceived Deficits Questionnaire (PDQ) scores. The average scores for the SF-36 HT, MFIS and PDQ between the 3 age groups are shown in Table 5. The youngest group of MS patients indicated a better change in health status over the last year compared to the oldest group (1.60 vs. $2.88, P$-value $=<0.05)$, the oldest group indicated a greater impact of fatigue compared to the youngest and mid-aged groups ( 2.88 vs. 1.60 vs. $2.22, P$-value $=0.035)$ and the oldest group indicated a greater perceived cognitive impairment compared to the mid-age group (37.33 vs. $18.22, P$-value $=0.027$ ). Additionally, no statistically significant differences in the EDSS scores of the 3 age groups were found indicating that the statistically significant differences found are independent of the severity of their disability. The average EDSS score for the youngest, mid and oldest age groups were 3.00, 4.94 , and 4.67 respectively. There were also no other

Table 4 Average SF-36 between 2 EDSS groups

\begin{tabular}{llll}
\hline Outcome & EDSS $\leq 3$ & EDSS $>5.5$ & EDSS $>5.5$ \\
\hline SF-36 PCS & 43.56 & 33.21 & $\mathbf{3 3 . 2 1}$ \\
SF-36 HT & 1.56 & 2.80 & $<\mathbf{0 . 0 5}$
\end{tabular}

Abbreviations: SF-36 36-item short form generic health status questionnaire, EDSS Kurtzke Expanded Disability Status Scale, PCS physical components summary scale, $H T$ health transition item

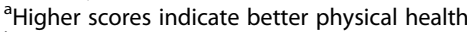

${ }^{\text {b}}$ SF-36 HT scale: 1, much better, 2, somewhat better, 3, same, 4, somewhat worse, 5, much worse

Captured in bold is $P=<0.05$ statistically significant differences in the remaining MSQLI mean scores.

\section{MSQLI and sex}

There were no statistically significant differences in the mean MSQLI scores between females and males. Additionally, the average EDSS for females was 3.75 and 4.39 for males, which was also not statistically significant.

\section{Discussion}

In this study, we evaluated the MSQLI data of minority MS patients to identify demographic and disease characteristics significantly associated with the quality of life of these patients residing in a large urban area. We found significant racial/ethnic differences in the HRQoL of people with MS. In this study, Hispanic White MS patients reported a worse overall mental health score compared to Non-Hispanic African Americans. More specifically, Non-Hispanic African Americans were found to have lesser anxiety and better behavioral control compared to Hispanic Whites based on selfreport. Hispanic Whites also reported a statistically significantly lower number of comorbidities than Non-Hispanic African Americans. Several studies have reported that physical and mental comorbidities negatively affect HRQoL [16-18]. However, hypertension was the only comorbidity reported by Hispanic Whites and little is known about the relationship between hypertension and HRQoL in MS patients and is often physically asymptomatic [19]. Additionally, both groups reported similar averages of EDSS scores and MS disease duration. No statistically significant differences were found in the use of illicit drugs or interferon medications as these can impact HRQoL as well. Racial and ethnic minorities in general tend to have poorer mental health, barriers to health, and receive lower quality mental health care [20]. However, despite sharing similar socio-demographic backgrounds, MS disease duration, and disability levels, the Hispanic MS patients perceived having worse psychological functioning. Our results suggest a health disparity in the mental health of Hispanics with MS. This may be related to language barriers and/or cultural differences in health behaviors and health attitudes. Language barriers are a major constraint in the treatment and care of Hispanics in general [21]. Additionally, in Hispanic culture there is a perception that mental health services are only for people who are severely disturbed and therefore seeking mental health services can be a stigma rather than a resource $[22,23]$. Future research is needed to analyze the mental health of Hispanics and African Americans with MS especially since the population of Hispanics in the U.S with MS will increase as the total Hispanic population increases. 
Table 5 Average MSQLI between 3 Age Groups

\begin{tabular}{lllll}
\hline Outcome & Born 1980-1994 (youngest) & Born 1965-1979 (middle-aged) & Born 1950-1964 (oldest) & $P$-value \\
\hline SF-36 HT & 1.60 & 2.22 & 2.88 & $<\mathbf{0 . 0 5}$ \\
MFIS $^{b}$ & 22.60 & 21.44 & 46.00 & $\mathbf{0 . 0 3 5}$ \\
PDQ $^{c}$ & 24.67 & 18.22 & 37.33 & $\mathbf{0 . 0 2 7}$ \\
\hline
\end{tabular}

Abbreviations: MSQLI multiple sclerosis quality of life inventory, SF-36 36-item Short Form Generic Health Status Questionnaire, HT health transition item, MFIS modified fatigue impact scale, $P D Q$ perceived deficits questionnaire

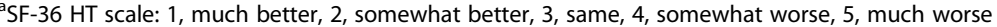

${ }^{b}$ Higher scores indicate greater impact of fatigue

'Higher scores indicate greater perceived cognitive deficits

Captured in bold is $P=<0.05$

We also found that MS patients with mild disability (EDSS $\leq 3)$ reported a better overall physical health and a better change in health status over the last year compared to those of moderate-severe disability (EDSS $>5.5$ ). These expected findings validate the EDSS because the EDSS measures disability on a scale of $0-10$ with higher scores indicating a higher level of disability so with scores $\leq 3$ one would anticipate patients with lower disability levels to report better physical health measures and better changes in health status. The youngest group (ages 23-37) of MS patients reported a better change in health status over the last year compared to the oldest group (ages 5367), the oldest group of MS patients reported a greater impact of fatigue compared to all age groups (ages 38-67) and lastly that the oldest group of MS patients reported a greater perceived cognitive impairment compared to the mid-aged group (ages 38-52). The youngest group (ages 23-37) also had the lowest average EDSS score (EDSS = 3) of all age groups. Moreover, the oldest group (ages 53-67) was found to have the most fatigue impact. Fatigue is the most common physical symptom reported by MS patients [24]. In a pilot study of Latinos with MS, fatigue was found to have the greatest impact on daily activities [21]. These results can be explained by the fact that with older age comes more fatigue. In addition, the oldest group (ages 53-67) indicated more perceived cognitive impairment compared to the mid-aged group (ages 38-52). No significant MSQLI findings were found between females and males. According to our results, the MSQLI and EDSS are independent valid measures of disability in our cohort.

The EDSS may be the most common outcome measure of impairment/disability used by MS specialists and neurologists, however it has limitations [6, 25]. For one, it is biased towards mobility [6]. Second, there are issues with inter-rater reliability in the minimal- moderate range of disability [26]. Third, physical function is only one aspect of a patient's experience $[27,28]$. In a quality of life study of MS patients, Göksel Karatepe and colleagues found that disability was negatively correlated with physical and mental health status in MS patients [29]. The EDSS is and should be complemented by measures of HRQoL [30]. HRQoL measures reflect the key determinants and concerns of a patient's overall health status from the patient's perspective [30]. It can detect the subtle disease-specific changes in MS that the EDSS cannot [31]. The MSQLI was selected as the measure of HRQoL in this study because of its comprehensiveness and specificity to MS patients. In a side to side comparison of 3 different MS-specific HRQoL measures, the MSQLI was shown to be more comprehensive in its coverage of the common MS symptoms [14]. Additionally, the MSQLI has been shown to work effectively in a field test with 300 North American patients with MS and a broad range of physical impairment (EDSS $=0-8.5$ ) [14]. It is continuously being used as an outcome measure in clinical trials [30, 32] and has been validated for use in older MS adults and cognitively impaired MS adults [33, 34]. Most importantly, it is well documented that patients with MS have a poorer quality of life than the healthy population and people with other chronic diseases [6, 29, 35-37]. This study provides added insight into how MS impacts and affects the HRQoL of minority patients with MS living in the United States.

However, this study has some limitations. Our sample size was small. There may have been a loss of translation in the Spanish version of the MSQLI because we translated the MSQLI into Spanish. However, others have translated different scales with success. For example, the quality of life in neurological disorders (Neuro-QoL) measurement system is another HRQoL instrument for adults and children with neurological disorders [38]. It was originally developed in English and shortly after translated to Spanish [38]. In a paper analyzing the Spanish version of the Neuro-QoL, Correia and colleagues concluded that both the adult and pediatric Spanish items were considered conceptually equivalent to the English source [38]. In a similar study, Fernandez and colleagues investigated the validity and reliability of the Spanish version of the Multiple Sclerosis International Quality of Life (MusiQoL) questionnaire [39]. They concluded the Spanish version of the MusiQoL questionnaire was a valid and reliable instrument for measuring quality of life in patients with MS in Spain [39]. Lastly, the type of MS medication may impact 
HRQoL. For instance, a patient who has to inject their medication on a daily basis may differ in their HRQoL compared to a patient with oral treatment. The study was not powered for this comparison.

\section{Conclusions}

This study uniquely compared the quality of life analysis of two ethnic populations, African Americans and Hispanics with MS at a large urban safety net hospital. Our study highlights the importance of the evaluation of quality of life in minority MS patients and the need for future research to investigate the mental health disparity "of and between" minorities with MS. Overall, physicians treating minority patients with MS should understand how MS symptoms affect different ethno-racial groups in order to provide culturally competent care to improve their quality of life.

\section{Acknowledgements}

The authors would like to thank the Conrad N. Hilton Foundation for grant funding associated with the project. All the authors have no other relevant disclosures.

\section{Funding}

Was provided by the Conrad N. Hilton Foundation.

\section{Availability of data and materials}

Data from the study will remain available for minimum of 3-10 years.

\section{Authors' contributions}

DG writing manuscript, research design, data collection, JL database maintenance, data collection, data review, manuscript review, KB database maintenance, data collection, data review, manuscript review, SV database maintenance, data collection, data review, manuscript review, ET database maintenance, data collection, data review, manuscript review, BM Primary Investigator, writing manuscript, research design, data collection and analysis. All authors read and approved the final manuscript.

\section{Ethics approval and consent to participate}

We received IRB approval for the study and consent was obtained from the patients.

\section{Consent for publication}

Study participants and authors were notified of this research would be published and deindentified results shared.

\section{Competing interests}

The authors declare that they have no competing interests.

\section{Publisher's Note}

Springer Nature remains neutral with regard to jurisdictional claims in published maps and institutional affiliations.

Received: 29 August 2018 Accepted: 13 December 2018 Published online: 17 January 2019

\section{References}

1. Calabresi PA. Diagnosis and management of multiple sclerosis. Am Fam Physician. 2004;70:1935-44

2. Noseworthy JH, Lucchinetti C, Rodriguez M, et al. Multiple sclerosis. N Engl J Med. 2000;343:938-52.

3. Poppe A, Wolfson C, Zhu B. Prevalence of multiple sclerosis in Canada: systematic review. Can J Neurol Sci. 2008:35:593-601.
4. Thompson AJ, Toosey AT, Ciccarelli O. Pharmacological management of symptoms in multiple sclerosis: current approaches and future directions. Lancet Neurol. 2010;9:1182-99.

5. Lublin FD, Reingold SC. Defining the clinical course of multiple sclerosis: results of an international survey. National Multiple Sclerosis Society (USA) advisory committee on clinical trials of new agents in multiple sclerosis. Neurology. 1996;46:907-11.

6. Nortvedt MW, Riise T, Myhr KM, et al. Quality of life in multiple sclerosis: measuring the disease effects more broadly. Neurology. 1999;53:1098-103.

7. Buchanan RJ, Huang C, Chakravorty BJ. Health-related quality of life among African Americans with multiple sclerosis. Ethn Dis. 2011;1:337-84.

8. Compston A. The genetic epidemiology of multiple sclerosis. Philos Trans $R$ Soc Lond Ser B Biol Sci. 1999:354:1623-34.

9. Kurtzke JF, Beebe GW, Norman JE Jr. Epidemiology of multiple sclerosis in U.S. veterans: 1. Race, sex, and geographic distribution. Neurology. 1979:29:1228-35.

10. Khan O, Williams MJ, Amezcua L, et al. Multiple sclerosis in US minority populations: clinical practice insights. Neurol Clin Pract. 2015:5:132-42.

11. U.S. Census Bureau (2016). Population estimates of the United States, July 1, 2016 Retrieved from https://www.census.gov/quickfacts/fact/table/US/ PST045216

12. U.S. Census Bureau (2014). National Population Projections, Table 10 Retrieved from https://www.census.gov/data/tables/2014/demo/popproj/ 2014-summary-tables.html

13. Ritvo PG, Fischer JS, Miller DM, et al. Multiple sclerosis quality of life inventory: a user's manual. National Multiple Sclerosis Society. 1997. https:// www.nationalmssociety.org/For-Professionals/Researchers/Resources-forResearchers/Clinical-Study-Measures/Multiple-Sclerosis-Quality-of-LifeInventory-(MSQL

14. Fischer JS, LaRocca NG, Miller DM, et al. Recent developments in the assessment of quality of life in multiple sclerosis. Mult Scler. 1999;5:251-9.

15. De Jong $H J I$, Kingwell $E$, Shirani $A$, et al. Evaluating the safety of $\beta$ interferons in MS: a series of nested case-control studies. Neurology. 2017; 88(24):2310-20

16. Turpin KV, Carroll $\amalg$, Cassidy JD, et al. Deterioration in the health-related quality of life of persons with multiple sclerosis: the possible warning signs. Mult Scler. 2007:13:1038-45.

17. Warren S, Turpin KV, Warren KG. Health-related quality of life in MS: issues and interventions. Can J Neurol Sci. 2009:36:540-1.

18. Berrigan L, Fisk J, Patten $S$, et al. Health-related quality of life in multiple sclerosis: direct and indirect effects of comorbidity. Neurology. 2016;86: $1417-24$

19. Warren SA, Turpin KVL, Pohar SL, et al. Comorbidity and health-related quality of life in people with multiple sclerosis. Int J MS Care. 2009;11:6-16

20. Mallinger JB, Lamberti JS. Psychiatrists' attitudes toward and awareness about racial disparities in mental health care. Psychiatr Serv. 2010;61:173-9.

21. Buchanan RJ, Zuniga MA, Carrillo-Zuniga G, et al. A pilot study of latinos with multiple sclerosis: demographic, disease, mental health, and psychosocial characteristics. J Soc Work Disabil Rehabil. 2011;10:211-31.

22. Alvidrez J. Ethnic variations in mental health attitudes and service use among low-income African American, Latina. and European American young women Community Ment Health J. 1999:35:515-30.

23. Interian A, Martinez IE, Guarnaccia PJ, et al. A qualitative analysis of the perception of stigma among Latinos receiving antidepressants. Psychiatr Serv. 2007;58:1591-4.

24. Putzki N, Katsarava Z, Vago $S$, et al. Prevalence and severity of multiplesclerosis-associated fatigue in treated and untreated patients. Eur Neurol. 2008:59:136-42.

25. Schwartz CE, Powell VE. The performance scales disability measure for multiple sclerosis: use and sensitivity to clinically important differences. Health Qual Life Outcomes. 2017:15:47.

26. Noseworthy $\mathrm{JH}$, Vandervoort MK, Wong CJ, et al. Interrater variability with the expanded disability status scale (EDSS) and functional systems (FS) in a multiple sclerosis clinical trial. Neurology. 1990;40:971-5.

27. Noble JG, Osborne LA, Jones KH, et al. Commentary on 'disability outcome measures in multiple sclerosis clinical trials. Mult Scler. 2012;18:1718-20.

28. Baumstarck $K$, Boyer $L$, Boucekine $M$, et al. Measuring the quality of life in patients with multiple sclerosis in clinical practice: a necessary challenge. Mult Scler Int. 2013:2013:524894.

29. Göksel Karatepe A, Kaya T, Günaydn R, et al. Quality of life in patients with multiple sclerosis: the impact of depression, fatigue, and disability. Int J Rehabil Res. 2011;34:290-8. 
30. Rudick RA, Miller D, Hass S, et al. Health-related quality of life in multiple sclerosis: effects of natalizumab. Ann Neurol. 2007;62:335-46.

31. Bandari DS, Vollmer TL, Khatri BO, et al. Assessing quality of life in patients with multiple sclerosis. Int J MS Care. 2010;12:34-41.

32. Cohen JA, Cutter GR, Fischer JS, et al. Benefit of interferon beta-1a on MSFC progression in secondary progressive MS. Neurology. 2002;59:679-87.

33. Dilorenzo T, Halper J, Picone MA. Reliability and validity of the multiple sclerosis quality of life inventory in older individuals. Disabil Rehabil. 2003;25:891-7.

34. Marrie RA, Miller DM, Chelune GJ, et al. Validity and reliability of the MSQL in cognitively impaired patients with multiple sclerosis. Mult Scler. 2003;9: $621-6$.

35. Hermann B, Vickrey B, Hays RD, et al. A comparison of health-related quality of life in patients with epilepsy. diabetes and multiple sclerosis. Epilepsy Res. 1996;25:113-8

36. Rothwell PM, McDowell Z, Wong CK, Dorman PJ. Doctors and patients don't agree: cross sectional study of patients' and doctors' perceptions and assessments of disability in multiple sclerosis. BMJ. 1997;314:1580-3.

37. Ford H, Gerry E, Johnson M, et al. Health status and quality of life of people with multiple sclerosis. Disabil Rehabil. 2001;23:516-21.

38. Correia H, Pérez B, Arnold B, et al. Spanish translation and linguistic validation of the quality of life in neurological disorders (neuro-QoL) measurement system. Qual Life Res. 2015;24:753-6.

39. Fernández $\mathrm{O}$, Fernández $\mathrm{V}$, Baumstarck-Barrau $\mathrm{K}$, et al. Validation of the spanish version of the multiple sclerosis international quality of life (musiqol) questionnaire. BMC Neurol. 2011;11:127.

Ready to submit your research? Choose BMC and benefit from:

- fast, convenient online submission

- thorough peer review by experienced researchers in your field

- rapid publication on acceptance

- support for research data, including large and complex data types

- gold Open Access which fosters wider collaboration and increased citations

- maximum visibility for your research: over $100 \mathrm{M}$ website views per year

At BMC, research is always in progress.

Learn more biomedcentral.com/submissions 